Review Article

\title{
Laryngotracheal injuries at a glance: review
}

\begin{abstract}
Objective: Laryngotracheal trauma is a rare but clinically important injury. These injuries may be accidental, suicidal or homicidal. Complications are frequent. Early recognition, accurate evaluation and proper treatment is essential to decrease morbidity among affected individuals and avoidance of complications.
\end{abstract}

Methods: Patients with external laryngotracheal injuries were analyzed retrospectively in a tertiary care centre. The outcome was assessed both in terms of voice and airway with regular follow up for 2years.

Results: The most common cause of injury is cut throat injuries followed by road traffic accidents. The main presenting symptoms and signs are hoarseness, haemoptysis, odynophagia and stridor in major injuries. Various sites of cut throat injury are of laryngeal framework i.e. thyroid cartilage, mixed soft tissue, cartilaginous injuries, thyrohyoid membrane, aryepiglottic fold and cricothyroid membrane. Patients with minor injuries, and patients with major injuries managed with early surgical intervention had better outcomes. Poor result was seen in patients with delayed surgical intervention.

Conclusion: Laryngotracheal trauma remains a clinically important injury requiring careful assessment with prompt recognition of injuries. Early surgical intervention with multidisciplinary approach is recommended for all major injuries to ensure a good outcome.
Volume 10 Issue I - 2018

\author{
Manya Thakur Roy \\ Devi Ahilya Vishwavidyalaya Indore, India
}

Correspondence: Manya Thakur Roy, Assistant Professor, Devi Ahilya Vishwavidyalaya, Indore, 33/3 Gitanjali Nagar, Raipur CG, India,Tel 8349393238,Email docmanyat@gmail.com

Received: January 26, 2018 | Published: February 14, 2018

Keywords: laryngotracheal trauma, neck injuries, intervention, management

\section{Introduction}

Anterior cut throat injuries posses major challenge in its management due to involvement of various vital structures. External laryngeal trauma, which is often a part of head and neck trauma, is relatively uncommon injury estimated at approximately 1 in every 22,900 emergency room visits. ${ }^{1}$ The most common associated injuries with laryngeal trauma, seen are intracranial injuries (13\%), open neck injuries $(9 \%)$ and cervical spine fractures $(8 \%){ }^{2}$

Patients with minor laryngeal injuries do well with medical management alone, whereas those with the major injuries invariably need tracheostomy. The cases with major disruption of the cartilaginous or soft tissue framework of the larynx require surgical repair with rigid internal fixation or intraluminal stenting under general anaesthesia.

\section{Discussion}

Cut throat injuries are the commonest cause of laryngeal trauma. Patients will invariably require either medical or surgical intervention or both, depending on the severity of the injury sustained. Medical treatment includes voice rest, oral steroids, antibiotics and at least 24hours of close observation in an intensive care unit.

Surgical treatment consists of tracheostomy and laryngeal exploration. Anterior neck injuries presenting as cut throat emergencies may be fatal if major blood vessels of the neck are involved. Establishment of good airway is the priority with attempt to preservation of phonation, which eventually depends on the surgical management. Complications have been estimated to be as high as $13 \%$ to $17 \%$ with regard to chronic airway Obstruction $^{3}$ and $21 \%$ to $25 \%$ with regard to voice compromise in studies involving both penetrating and blunt laryngeal injury. ${ }^{4}$

Ocassionally, external trauma to the laryngotracheal complex in both the adult and pediatric population can lead to rapid asphyxiation, due to aspiration of blood, intrapulmonary haemorrhage, cricotracheal separation and/or recurrent laryngeal nerve palsy. In cases of external trauma, concomitant cervical spine injury CS (14\%), closed head injury (13-28\%), chest trauma (13\%), fractured ribs or sternum, pneumothorax, haemothorax, or disruption of the esophagus (3-14\%) may occur and should be included in the evaluation. ${ }^{5}$

There is need of multidisciplinary approach involving combined effort of otorhinolaryngologist, anesthesiologist and psychiatrist if suicidal injuries are sustained. Careful Endoscopic evaluation is often required to rule out concomitant injury to structures within the upper aerodigestive tract. Bubbling of air through neck wound indicates perforation of airway. The presence of retropharyngeal air seen in lateral view of $\mathrm{x}$-ray neck indicates perforation of either the pharynx or oesophagus. ${ }^{6}$

We generally do not advise tracheostomy in all patients having minor cut throat injuries with non-displaced thyroid cartilage fractures and minimal intralaryngeal injury (without air leak). Every case should be assessed based on the extent of the injury and structures involved, and availability of resources to cope up with future airway problems. Timely reconstruction of airway in layers based on adequate knowledge of Neck anatomy can give fair outcome. Similarly an advance in fibreoptic intubation and airway management has reduced considerably the need for routine tracheostomy for patients with airway problems. Bryce ${ }^{7}$ advocates use of one or two stainless steel wires in addition to absorbable suture to provide strength with head flexion position during postoperative period for 7 to 10 days by means of a suture from the chin to the sternum to relieve tension on the anastomosis. Whereas in reanastomosis of a transected trachea, most authors suggest the use of absorbable suture. A good outcome is said to be achieved when patient had a normal airway and can be decannulated without any difficulties with a good voice.

\section{Conclusion}

To conclude, early repair of mucosal and cartilaginous injury leads to improved functional outcome with a normal airway with no dysphagia and acceptable voice in majority of patients. Therefore, in 
a case of transected trachea securing airway by doing tracheostomy below the injury, just anastomosing and maintaining neck in flexion, will be adequate to allow tension free anastomosis, thus establishing secure airway with relatively simple surgical method, which can be done even in peripheral hospitals. Concurrent psychiatric assessment along with prompt meticulous layer by layer surgical repair within 24 hours of the injury and stepwise post operative management with Nasogastric tube is advocated to prevent long term complications like pharyngocutaneous fistula, aerodigestive tract stenosis.

\section{Acknowledgements}

None.

\section{Conflicts of interest}

Author declares there are no conflicts of interest.

\section{Funding}

None.

\section{References}

1. Kandogan T, Olgun L, Gültekin G, et al. External laryngeal trauma. Swiss Med Wkly. 2003;133(25-26):372.

2. Jewett BS, Shockley WW, Rutledge R. External laryngeal trauma analysis of 392 patients. Archives Arch Otolaryngol Head Neck Surg. 1999;125(8):877-880.

3. Kurien M, Zachariah N. External laryngotracheal trauma in children. Int J Pediatr Otorhinolaryngol. 1999;49(2):115-119.

4. Minard G, Kudsk KA, Croce MA, et al. Laryngotracheal trauma. Am Surg. 1992;58(3):181-187.

5. Le May, SR Le Malokiiiiy. Penetrating Wounds of the Larynx and Cervical Trachea. Archives of Otolaryngology. 1971;94(6):558-565.

6. Davies JR. The fibreoptic laryngoscope in the management of cut throat injuries. British journal of Anaesthesia. 1978;50(5):511-514.

7. Bryce DP. The Surgical Management Laryngotracheal Injury. The Journal of Laryngology \& Otology. 1972;86(6):547-558. 